Original research article

\title{
Cerebral vasomotor reactivity predicts the development of acute stroke in patients with internal carotid artery stenosis
}

\author{
Keju Ju, Lingling Zhong, Xiaoyu Ni, Hua Cao, Guanliang Cheng, \\ Lianshu Ding
}

Department of Neurology, Huai'an First People's Hospital, Nanjing Medical University, 6 Beijing Road West, Huai'an 223300, China

\section{A R T I C L E I N F O}

\section{Article history:}

Received 30 August 2017

Accepted 28 December 2017

Available online 8 January 2018

Keywords:

Vasomotor reactivity

Transcranial Doppler

Carotid artery stenosis

Cerebral infarction

Stroke

\begin{abstract}
A B S T R A C T
Objective: To investigate the relationship between cerebral vasomotor reactivity (VMR) and acute stroke in patients with internal carotid artery stenosis.

Methods: 54 patients with internal carotid artery stenosis were enrolled. VMR was calculated by transcranial Doppler monitoring of the velocity of blood flow. 3-Dimensional dynamic contrast enhanced magnetic resonance angiography was used to detect stenosis, and diffusion weighted imaging was used to detect infarction.

Results: VMR value was significantly lower in patients with carotid artery stenosis than in control group ( $T=3.112, P=0.002$ ), and significantly lower in patients with aortic atherosclerotic stroke than in non-infarct group $(T=10.930, P=0.000)$. However, VMR value was significantly higher in patients with new-onset small-artery occlusion stroke than in noninfarction group $(T=-2.538, P=0.013)$. Scatter plots showed that aortic atherosclerotic stroke occurred mainly in patients with severe internal carotid artery stenosis, and VMR value in cerebral artery significantly decreased.

Conclusion: Decreased VMR value is an important prognostic factor for the occurrence of aortic atherosclerotic stroke, and can be used as a reference for preoperative hemodynamic evaluation in patients with internal carotid artery stenosis.

(c) 2018 Polish Neurological Society. Published by Elsevier Sp. z o.o. All rights reserved.
\end{abstract}

\section{Introduction}

Carotid stenosis is a progressive narrowing of carotid artery due to the buildup of plaque (atherosclerosis) inside the artery that reduces blood flow to the brain. Carotid artery stenosis increases the risk of atherosclerotic stroke because of plaque deposits. In recent years, more surgical interventions of internal carotid artery stenosis have been reported. Among them, internal carotid artery endarterectomy and stenting are

\footnotetext{
* Corresponding author.

E-mail address: hajukeju@163.com (L. Ding).

https://doi.org/10.1016/j.pjnns.2017.12.015

0028-3843/@ 2018 Polish Neurological Society. Published by Elsevier Sp. z o.o. All rights reserved.
} 
the two main methods which significantly improve the prognosis of patients with internal carotid artery stenosis $[1,2]$. Currently, the main criteria for the selection of intervention or surgical treatment is the judgment of stenosis extent by imaging. Unfortunately, many patient with severe stenosis or even occlusion may not have clinical symptoms.

At present, the detection of cerebral blood flow can be carried out by single photon emission computed tomography (PET-CT), isotope perfusion imaging (SPECT), CT perfusion imaging (CTP), and magnetic resonance perfusion imaging (PWI), DSA angiography, and transcranial Doppler (TCD). Cerebral vasomotor reactivity (VMR) test is a method to detect the changes in cerebral blood flow velocity with changes in $\mathrm{CO}_{2}$ partial pressure in vivo by TCD. VMR can reflect cerebrovascular reserve (CVR), indirectly reflect collateral circulation compensation, and provide a new evaluation criterion for the selection of surgery or intervention [3]. Currently, the relationship between VMR and the development of acute stroke in patients with internal carotid artery stenosis has been investigated but the reported data are controversial.

This study aimed to investigate the relationship between cerebral VMR and acute stroke in patients with internal carotid artery stenosis.

\section{Subjects and methods}

\subsection{Patients}

This study enrolled 54 patients hospitalized from March 2016 to November 2016, involving 107 carotid arteries (one case had no temporal window on one side). Among them, 25 cases were acute infarction, 3 cases were transient ischemic attack, 3 cases were cardiogenic cerebral embolism, 23 cases were dizziness, headache and other diseases. There were 33 males and 21 females, with an average age of $59.50 \pm 12.66$ years. The study was approved by Institute Ethics Committee and all patients provided informed consent.

\subsection{VMR measurement}

VMR was measured using Viasys sonar Digital TCD. Two Doppler $2 \mathrm{MHz}$ probes were fastened to the head over the temporal window, and the ipsilateral middle cerebral artery was chosen as the target vessel. The middle cerebral artery was further identified by compression neck test. The mean velocity $\left(V_{m}\right)$ in normocapnia $\left(V_{m}\right.$ Normo) and in hypercapnia $\left(V_{m}\right.$ Hyper) was determined and $V_{m}$ change curve was drawn. VMR was calculated as follows: $\left(V_{m}\right.$ hyper $-V_{m}$ normo $) / V_{m}$ normo) $\times 100 \%$.

\subsection{Detection of vascular stenosis}

3-Dimensional dynamic contrast enhanced magnetic resonance angiography (3D DCE-MRA) was used to detect vascular stenosis. The stenosis rate of the blood vessel was calculated as (the diameter of the stenotic distal normal blood vessel - the diameter of the stenosis)/the diameter of the stenotic distal normal blood vessel) $\times 100 \%$. For patients with multiple stenosis, the most severe stenosis part was defined as the stenosis rate of the blood vessel. According to the presence of stenosis, the patients were divided into carotid artery stenosis group and non-stenosis group, and the difference in VMR between the two groups was compared.

\subsection{The classifications of acute infarction}

All patients underwent craniocerebral MRI within three days of admission. The presence of acute infarction was determined based on the detection of high signal by carotid artery diffusion-weighted imaging (DWI). Based on clinical history, the symptoms of patients and the imaging, the patients were divided into: no cerebral infarction (NCI), large artery atherosclerosis-no artery embolism (LA-NE), large artery atherosclerosis-artery embolism (LA-E), small artery occlusion lacunar (SA), cardio embolism and other (CE). The differences in VMR among different types of acute infarct and non-infarct groups were compared.

\subsection{Statistical analysis}

All data were analyzed using SPSS Statistics 17.0 software. Ttest was used to compare VMR values among different groups, and the scatter plot was drawn. $P<0.05$ indicated significant difference.

\section{Results}

VMR value was significantly lower in patients with carotid artery stenosis than in control group $(T=3.112, P=0.002)$, the difference was statistically significant (Table 1). Comparison of corresponding arterial areas in different types of fresh infarcts with non-infarction showed that VMR value was significantly lower in patients with atherosclerotic stroke than in those without infarction ( $T=10.930, P=0.000$ ). However, VMR value was significantly higher in patients with new-onset small artery occlusion stroke than in non-infarction group ( $\mathrm{T}=-2.538, \mathrm{P}=0.013)$. There was no significant difference in VMR of arterial-arterial embolism group and cardiogenic cerebral embolism group, compared to non-infarction group (Table 2).

Table 1 - Comparison of VMR in carotid artery stenosis and non-stenotic vessels.

\begin{tabular}{lccccc} 
& $N$ & Mean & Standard deviation & $T$ & $P$ \\
\hline Non-carotid artery stenosis & 59 & 0.579 & 0.210 & 3.112 \\
Carotid artery stenosis & 48 & 0.433 & 0.264 & 0.002 \\
\hline
\end{tabular}


Table 2 - Comparison of VMR in different cerebral infarction types and non-infarct vessels.

\begin{tabular}{|c|c|c|c|c|c|}
\hline Infarction type & $N$ & Mean & Standard deviation & $\mathrm{T}$ & $P$ \\
\hline NCI & 75 & 0.534 & 0.228 & & \\
\hline LA-NE & 10 & 0.157 & 0.071 & 10.930 & 0.000 \\
\hline LA-E & 11 & 0.516 & 0.204 & 0.250 & 0.803 \\
\hline SA & 8 & 0.747 & 0.202 & -2.538 & 0.013 \\
\hline $\mathrm{CE}$ & 3 & 0.536 & 0.184 & -0.020 & 0.986 \\
\hline
\end{tabular}

No cerebral infarction (NCI), large artery atherosclerosis-no artery embolism (LA-NE), large artery atherosclerosis-artery embolism (LA-E), small artery occlusion lacunar (SA), cardio embolism and other (CE).

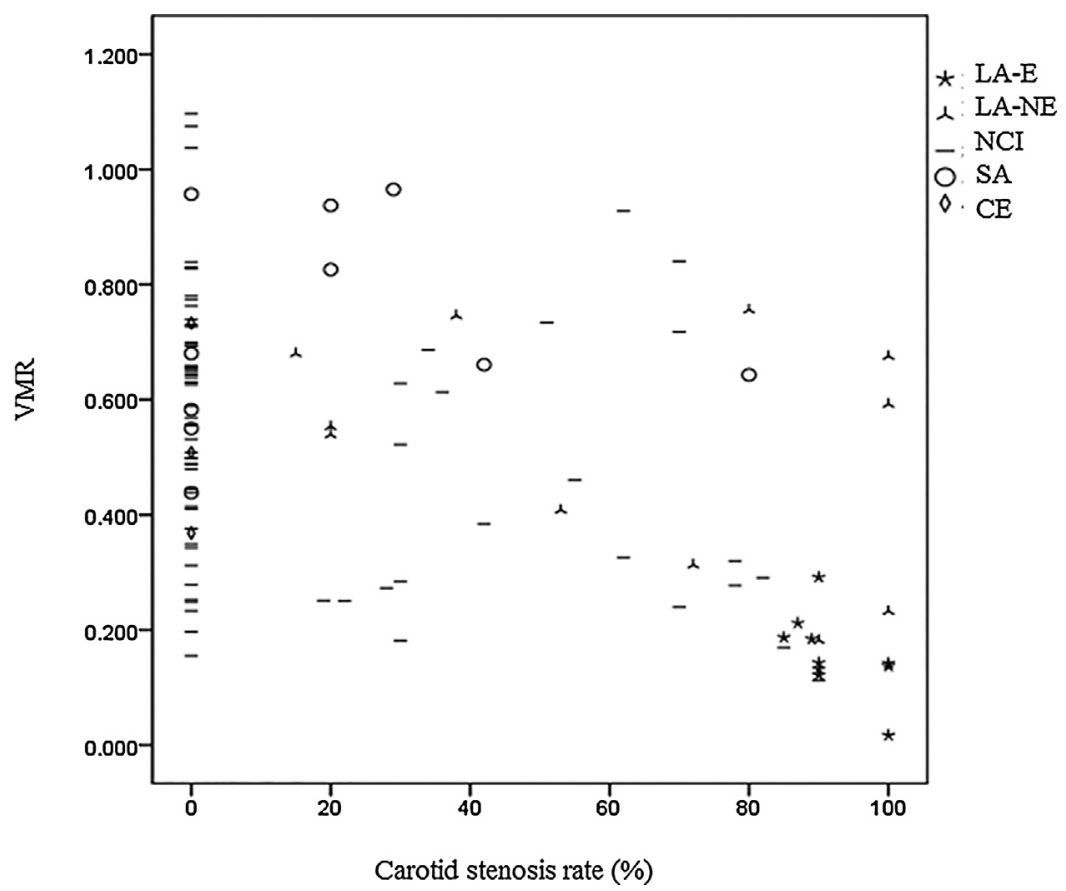

Fig. 1 - The distribution of VMR in patients with internal carotid artery stenosis and different types of cerebral infarction: no cerebral infarction (NCI), large artery atherosclerosis-no artery embolism (LA-NE), large artery atherosclerosis-artery embolism (LA-E), small artery occlusion lacunar (SA), cardio embolism and other (CE).

Scatter plot analysis showed that aortic atherosclerotic stroke mainly occurred in patients with severe internal carotid artery stenosis (stenosis rate $>80 \%$ ), while VMR value of corresponding cerebral artery was significantly lower $(<0.30)$. Arterial thrombosis of large arterial sclerosis was distributed in the entire carotid artery stenosis area, and VMR value had no aggregation characteristics. Small artery stenosis was mainly distributed in patients with no obvious internal carotid artery stenosis or mild stenosis, and VMR value was high $(>0.40)$. Cardiogenic cerebral embolism and other patients did not show obvious characteristics (Fig. 1).

\section{Discussion}

Under physiological conditions, the changes in cerebral perfusion pressure are influenced by blood pressure, intracranial pressure, vascular resistance and other factors. When cerebral perfusion pressure changes, the vessels will expand or contract to maintain a constant cerebral blood flow to ensure normal cerebral metabolism [4]. However, in pathological conditions such as carotid artery or cerebral artery stenosis, the narrow blood vessels can lead to significant decrease in distal perfusion pressure, resulting in extreme expansion of blood vessels to adapt to metabolic needs of the brain. When the extreme expansion of blood vessels cannot maintain metabolic needs, the symptoms of ischemia will occur, leading to brain necrosis and cerebral infarction.

VMR measurement employs the principle that metabolic carbon dioxide can stimulate cerebral vasodilatation to determine cerebrovascular reserve expansion. $\mathrm{CO}_{2}$ inhalation test to assess VMR was safe and feasible, and the results had a good correlation with functional magnetic resonance and PET brain perfusion studies [5,6]. In patients with asymptomatic carotid stenosis, decreased VMR was often associated with impairment of cognitive function and leukoencephalopathy [7-9]. However, in patients with symptomatic carotid stenosis, decreased vascular reactivity reserve and decreased oxygen uptake were significantly 


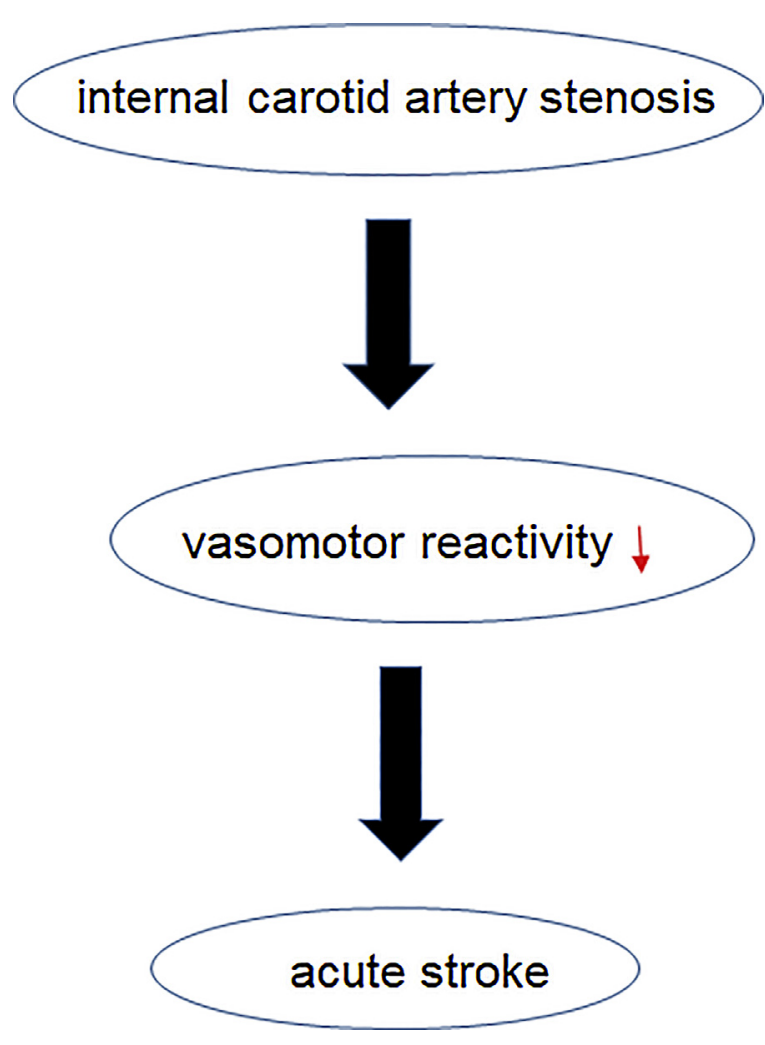

Fig. 2 - The relationship between cerebral vasomotor reactivity, internal carotid artery stenosis and acute stroke. After internal carotid artery stenosis, cerebral vasomotor reactivity will reduce due to the presence of atherosclerotic plaque. Reduced vasomotor reactivity will increase the risk of acute atherosclerotic stroke.

associated with stroke [10]. VMR can be improved in patients after successful carotid surgery [11].

In this study we showed that VMR value in patients with carotid artery stenosis, especially in patients with severe stenosis, was significantly lower than in control. The scatter plot showed that VMR was below 30\% in new-onset acute infarction patients who also had higher carotid stenosis rate of more than $80 \%$, consistent with the study of Puz et al. [12]. These data indicate that the compensation of blood vessels in these patients after stenosis is insufficient, leading to vascular reactivity failure. Therefore, the treatment of these patients should mainly focus on the plaques. In this study, VMR increased in patients with arterial lesions compared with patients without infarction, the reason may be related to the bias of case selection.

Internal carotid artery stenosis is a clinically common lesion of blood vessel leading to ischemic stroke, and is also the most commonly selected target vessel for surgical or interventional treatment. However, due to the difference of posterior collateral circulation compensation, preoperative multimodal hemodynamics assessment has been paid more attention [13]. VMR measurement is one of the simple and effective methods. We believe that the decline of VMR reflects the failure of the distal vessel to compensate, and thus is an important predictor of ischemic events (Fig. 2). Despite the limitations of this study such as small sample size, our results suggest that VMR could be used as an important reference for preoperative hemodynamic assessment and screening of patients with carotid stenosis.

\section{Conflict of interest}

None declared.

\section{Acknowledgement and financial support}

This study was supported by the Science and Technology Development Foundation of Huai'an to Dr. Ding (No. HAS2015018).

\section{R E F E R E N C E S}

[1] Siddiq F, Adil MM, Malik AA, Qureshi MH, Qureshi AI. Effect of carotid revascularization endarterectomy versus stenting trial results on the performance of carotid artery stent placement and carotid endarterectomy in the United States. Neurosurgery 2015;77:726-32.

[2] Akinci T, Derle E, Kibaroglu S, Harman A, Kural F, Cinar P, et al. Clinical results of carotid artery stenting versus carotid endarterectomy. Neurosciences (Riyadh) 2016;21:319-25.

[3] Pandya A, Gupta A, Kamel H, Navi BB, Sanelli PC, Schackman BR. Carotid artery stenosis: cost-effectiveness of assessment of cerebrovascular reserve to guide treatment of asymptomatic patients. Radiology 2015;274:455-63.

[4] Wolf ME. Functional TCD: regulation of cerebral hemodynamics-cerebral autoregulation, vasomotor reactivity, and neurovascular coupling. Front Neurol Neurosci 2015;36:40-56.

[5] Caputi L, Ghielmetti F, Farago G, Longaretti F, Lamperti M, Anzola GP, et al. Cerebrovascular reactivity by quantitative magnetic resonance angiography with a Co(2) challenge. Validation as a new imaging biomarker. Eur J Radiol 2014;83:1005-10.

[6] Persoon S, Kappelle LJ, van Berckel BN, Boellaard R, Ferrier $\mathrm{CH}$, Lammertsma AA, et al. Comparison of oxygen-15 PET and transcranial Doppler $\mathrm{CO}_{2}$-reactivity measurements in identifying haemodynamic compromise in patients with symptomatic occlusion of the internal carotid artery. EJNMMI Res 2012;2:30.

[7] Buratti L, Viticchi G, Falsetti L, Balucani C, Altamura C, Petrelli C, et al. Thresholds of impaired cerebral hemodynamics that predict short-term cognitive decline in asymptomatic carotid stenosis. J Cereb Blood Flow Metab 2016;36:1802-4.

[8] Han X, Ouyang L, Zhang C, Ma H, Qin J. Relationship between deep medullary veins in susceptibility-weighted imaging and ipsilateral cerebrovascular reactivity of middle cerebral artery in patients with ischemic stroke. Exp Ther Med 2016;11:2217-20.

[9] Zavoreo I, Basic Kes V, Lisak M, Marsic N, Ciliga D, Trost Bobic T. Cognitive decline and cerebral vasoreactivity in asymptomatic patients with severe internal carotid artery stenosis. Acta Neurol Belg 2013;113:453-8. 
[10] Pindzola RR, Sashin D, Nemoto EM, Kuwabara H, Wilson JW, Yonas $\mathrm{H}$. Identifying regions of compromised hemodynamics in symptomatic carotid occlusion by cerebrovascular reactivity and oxygen extraction fraction. Neurol Res 2006;28:149-54.

[11] Telman G, Kouperberg E, Nitecki S, Karram T, Schwarz HA, Sprecher E, et al. Cerebral hemodynamics in symptomatic and asymptomatic patients with severe unilateral carotid stenosis before and after carotid endarterectomy. Eur J Vasc Endovasc Surg 2006;32:375-8.
[12] Puz P, Lasek-Bal A, Urbanek T, Kazibutowska Z. Assessment of cerebral embolism and vascular reserve parameters in patients with carotid artery stenosis. Neurol Neurochir Pol 2016;50:356-62.

[13] Sharma VK, Tsivgoulis G, Ning C, Teoh HL, Bairaktaris C, Chong VF, et al. Role of multimodal evaluation of cerebral hemodynamics in selecting patients with symptomatic carotid or middle cerebral artery stenoocclusive disease for revascularization. J Vasc Interv Neurol 2008;1:96-101. 